\title{
NEEDS AND OPPORTUNITIES FOR NANOTRIBOLOGY IN MEMS AND NEMS
}

\author{
R.W. Carpick $^{l^{*}}$, G.E. Wabiszewski ${ }^{l}$,F. Streller ${ }^{2}$
}

${ }^{1}$ Dept. of Mechanical Engineering and Applied Mechanics, U. Pennsylvania, Philadelphia, Pennsylvania, USA

${ }^{2}$ Dept. of Materials Science and Engineering, U. Pennsylvania, Philadelphia, Pennsylvania, USA

\begin{abstract}
Nanoelectromechanical systems (NEMS) switches are a candidate "beyond CMOS" technology, with a key benefit being massively reduced power consumption. However, the reliability of the contact interface is a principal challenge, as the electrically conducting contacting surfaces need to be able to open and close up to a quadrillion times without excessive adhesion, wear, or contamination. Similar failure mechanisms occur in microelectromechanical systems (MEMS). These failure mechanisms are not well understood, and materials that can exhibit the needed performance have not yet been demonstrated. Here we highlight key challenges and opportunities for applying scientific insights from nanotribology to address these reliability challenges.
\end{abstract}

\section{INTRODUCTION}

Nanoelectromechanical systems (NEMS) switches have been identified as a potential next-generation transistor with far lower power consumption than existing electronic integrated circuits, a critical technological need. These switches are nanoscale moving devices that convert an electrical input signal into motion to close a conductive contact [1]. NEMS switches are thus a mechanical version of a transistor with topologies that often mimic larger microelectromechanical systems (MEMS) switches. However, NEMS switches require small dimensions for the fast, competitive switching speeds $(<100 \mathrm{~ns})$ desired in computer logic applications. The nanoscale dimensions and complex operating conditions at the electrical contact make NEMS logic switches susceptible to tribologically-mediated failure mechanisms. In particular, low contact and restoring forces may lead to device "stiction" (device permanently stuck closed), or intolerable increases in switch contact resistance due to the formation of insulating tribopolymer (TP) films. It may be possible to mitigate these failures with the use of low adhesion and catalytically inactive contact materials. This paper will review the opportunities presented by NEMS switch technology, and will discuss needs and approaches to address the technological barriers arising from tribological issues.

\section{The Need for Low Power Computation}

Sustained growth in computing power and decrease in computing cost has led to the proliferation of devices utilizing integrated processors in the last half century. These processors are overwhelmingly based on fully-electronic CMOS technology. While this technology has proven exceptional for decreasing transistor real estate and increasing speed [2], it is currently encountering a "power crises." Further scaling of CMOS leads to intractable increases in power loss per computation due to irreversible processes inherent to the physics controlling device operation [2]-[4]. The significant power requirements of computer processors, the power crisis of conventional CMOS, trends towards smart devices, the increasing penetration of home and laptop computers, the desire for long lasting battery-operated mobile devices, and power consumption requirements of CMOS outpacing battery capacities motivates the need to explore lower power transistors. Recognizing the physical limitations of existing CMOS technology, the International Technology Roadmap for Semiconductors (ITRS) has included nanoelectromechanical systems (NEMS) switches as a possible disruptive, low-power technology to cohabitate or usurp the conventional, fully electronic transistor.

\section{NEMS Switches as an Alternative to the Transistor}

Ohmic NEMS switches provide the same functionality as the conventional metal-oxide-semiconductor field-effect transistor (MOSFET). In both technologies, the application of a gate signal results in current transfer from the source to drain. However, CMOS relies on a fully-electronic, semiconductor junction to achieve this functionality whereas NEMS logic switches rely on the mating of conductive contacts (see Figure 1).

NEMS ohmic switches utilize mechanical motion to modulate the distance between two conductive contacts which serve as the source (S) and drain (D) electrodes. Figure 1(a) shows the topology and working principal of a NEMS switch prototype by Piazza and co-workers [5], [6]. The device is in an "off-state" when the source and drain electrodes are separated by a physical gap, and the "on-state" when the source and drain electrodes are closed with sufficient force to establish an electrical connection. The space separating the electrodes in the off-state is the switch gap. Ideally, closure of the source and drain electrodes results in current flow from the biased source to the drain, while contact release results in a near-infinitely resistive junction. Successful operation is dependent on reliably and repetitively making and breaking the source and drain electrodes while maintaining a conductive contact in the device on-state and a high resistance in the off-state.
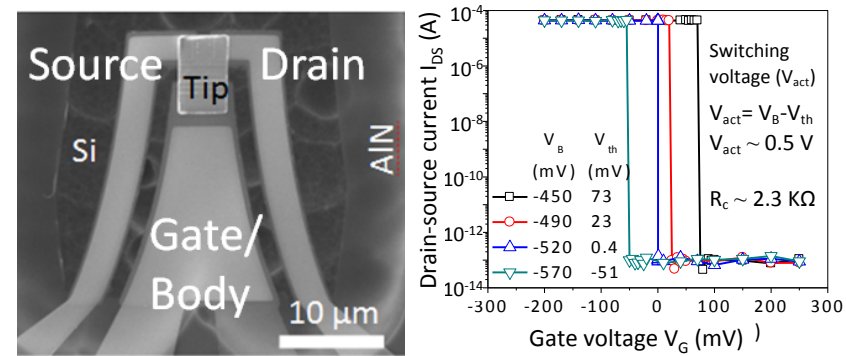

Figure 1. (a) SEM top view image of a working NEMS switch. (b) The device shows a steep subthreshold slope, low leakage current, and low switching voltage $\left(V_{B}=\right.$ switch body bias voltage, $V_{\text {th }}=$ gate threshold voltage for switching, $V_{\text {act }}=$ total actuation voltage, $R_{C}=$ contact resistance). From [6].

An input voltage, the gate $(\mathrm{G})$ signal, is converted to mechanical motion to modulate the gap. This motion can be achieved through various means of transduction using many topologies. Ohmic NEMS switches utilizing electrostatic [7]-[9] and piezoelectric [5], [6] actuation employing a range of geometries (see review in [10]) have been demonstrated. Regardless, all ohmic NEMS switches depend on reliable closure and separation of a conductive interface to achieve switching.

CMOS faces a scaling and power crisis. Continued Moore's Law scaling has contributed to increasing device leakage, resulting in significant power loses even when switching is not occurring [11]. Furthermore, a breakdown of Moore's law is predicted by approximately 2020 as critical MOSFET dimensions exceed physical limitations [2], [12]. Ohmic NEMS logic switches that reduce power draw per computation have been identified in the ITRS as a potential next-generation technology to cohabitate or usurp FETs [2]. Comparisons between NEMS relay-based logic to conventional CMOS revealed that energy savings of one to three orders of 
magnitude may be achieved with NEMS relays due to the low power consumption at the individual switch level [13]-[15] and design advantages unique to mechanical relays that reduce the number of switches necessary to perform logic operations [16]. The reduced power consumption is primarily a consequence of the physics underpinning device operation [17]. Figure 1(b) demonstrates the potential power savings via the steep on-off slope and the low gate voltage, as low as $0.4 \mathrm{mV}$ through use of a body bias. The power consumption of a digital switch is characterized by power dissipated during both the off-state $\left(V_{\mathrm{G}}<0\right.$, sub-threshold leakage) and on-state $\left(V_{\mathrm{G}}>0\right.$, dynamic switching power). NEMS logic switches afford power savings both regimes [18], [19].

Off-state leakage in CMOS is dominated by source-drain and gate leakage that scales unfavorably with decreasing device dimensions [17]. This is seen as a current offset for voltages below the turn on threshold voltage. This leakage is due shorter and thinner oxide gate channels necessary to continue Moore's Law scaling. Sub-threshold leakage in CMOS currently represents $\sim 50 \%$ of the total microprocessor power density [17] with standby leakage currents of $1 \mathrm{nA} /$ transistor having been reported at $250 \mathrm{~nm}$ gate-width node. This leakage is dominated by gate leakage through the thin gate oxide and continues to increase with device downscaling. NEMS logic relays have already demonstrated leakage values five orders lower than MOSFET at the single device level [5]. These lower leakage values are due to the physical gap between the source and drain, eliminating source to drain leakage so long as the gap is $>2 \mathrm{~nm}$ [2]. Unique to mechanical relays, physical gaps and low leakage gate materials (in the case of piezoelectricallyactuated devices) reduce gate leakage to effectively zero.

NEMS relays also offer far lower dynamic switching power than CMOS. The sub-threshold swing describes the inverse slope of the I-V curve and determines the voltage needed to attain a decade change in source to drain current [20]. Lower sub-threshold swings correspond to lower turn-on voltages, which confer lower active transistor power consumption. CMOS sub-threshold swing is thermodynamically limited by electron drift and diffusion to 60 $\mathrm{mV} / \mathrm{dec}[11]$, with existing CMOS sub-threshold swings typically $70-100 \mathrm{mV} / \mathrm{dec}$ [17], [20]. This translates to turn on voltages of $0.3-1 \mathrm{~V}$ [21], several orders of magnitude larger than the minimum voltage (a few $\mathrm{mV}$ ) necessary for communication. NEMS prototype logic relays offer the lowest sub-threshold slope of all potential transistor replacement devices [17] with sub-threshold slopes of $0.03-2 \mathrm{mV} / \mathrm{dec}$ already demonstrated [5], [6], [22], translating to threshold voltages of just a few $\mathrm{mV} \mathrm{[6].}$

In addition, NEMS logic relays are amenable to application spaces not accessible by CMOS. The functionality of CMOS relies critically on delicate doping levels that impose strict thermal budgets and minimization of heat generation due to device leakage. As NEMS logic does not rely on doping levels, these devices may be amenable to high temperature computing, allowing sensors in previously inaccessible, harsh environments such as temperatures up to $500{ }^{\circ} \mathrm{C}$ [23], [24]. Furthermore, co-integration of NEMS relays with CMOS -possible due to the low thermal budget of typical NEMS processing [2] - has led to the exploration of programmable gate array logic based on NEMS relays and traditional MOSFET [25], [26]. The presence of physical gaps in NEMS relays also confers robustness against electromagnetic shocks, which makes these transistors attractive in military applications where radiationhard attributes are desired. Adhesion between the electrical contacts of NEMS relays may also be used for nonvolatile memory applications where the switch retains its last state (open or stuck closed) [27], [28]. NEMS relays are also amenable to three dimensional integration, which could increase functional density per given real-estate of a microchip to continue Moore's Law scaling.

\section{CONTACT: NEMS' "ACHILLES' HEEL"}

Despite the potential benefits of NEMS logic switches, tribological failure mechanisms at the electrical contact interface limit their viability [2]. Transistor functionality critically depends on maintaining high isolation in the off-state and low resistance in the on-state. Failure due to stiction that results in permanent welding of the switch interface, wear of contact materials, adsorbed layers of insulating contaminant films on free surfaces, and insulating tribopolymer (TP) formation of chemomechanical origin have been observed in microscale and nanoscale electrical contacts testing [10], [29]. The term tribopolymer (TP) is used in place of the more commonly used term "friction polymer". Friction polymers were first observed in sliding electrical contacts. However, it has since been observed that such polymer formation can be achieved under normal stresses (absence of significant shear stresses)[30].

The effects of stiction, insulating contaminant layers, and TP formation are expected to increase in severity as NEMS relays are scaled down in size. This is a consequence of the dominance of surface forces and the limited closure and separation forces available to NEMS relays. Figure 2(a) compares the closure and separation forces of micro- and nanoscale electrical switches and the necessary separation force for single (the fundamental unit of contact in nanoscale switches) to multiple contacting asperities indicative of multiasperity, microscale contacts. This approximation shows that the surface forces of NEMS relay contacts will outpace the relative generative force of the device. Microscale switches with soft, low hardness metallic electrical contacts (e.g. Au) have been particularly susceptible to stiction due to electromigration or softening that results in contact area growth [31]. Consequently, highly adhesive materials must be avoided to prevent stiction.
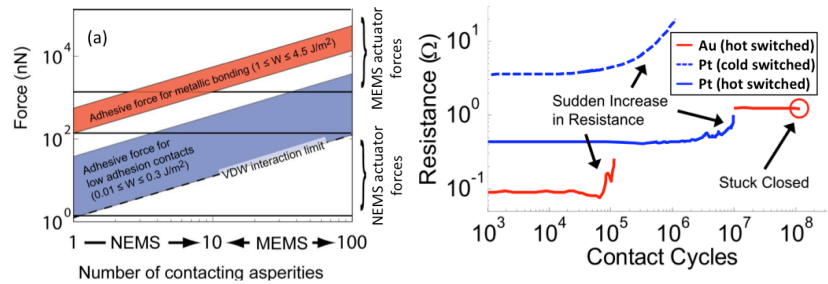

Figure 2. (a) Separation force of electrical contacts of various ranges of work of adhesion ranges, $W$, vs. the number of simultaneously interacting surface asperities. The asperity interactions are treated with adhesive contact mechanics. The adhesive force of nanoscale contacts can exceed the restoring force of typical NEMS actuators. (b) Contact resistance vs. make/break cycles for microscale electrical contacts of Au and Pt. TP formation causes dramatic increases in contact resistance.

Inhibiting the insulating TP formation may be the greatest challenge facing NEMS switches. It is affected by the gas environment, mechanical, electrical, and chemical properties of the contact materials, and the electrical power through the contacts. Figure 2(b) shows the effect of repetitive cycling of microscale noble metal contacts under both cold and hot cycling. Even for such non-reactive materials, contact resistance increases after $10^{5}$ to $10^{8}$ cycles, attributed to carbon and oxygen contaminants [29]. The origin of this effect appears to be mechanochemical - the presence of free surfaces, environmental contaminants, and pressure activate bond formation and chain lengthening. Even hermetically-sealed devices have demonstrated similar behavior [30]. Recent evidence suggests that operation of contacts in reducing environments such as oxygen can significantly reduce TP buildup [32] However, such device packaging can be costly to implement and limit the operation space of the device. Ultimately, conductive and non-reactive electrical contact materials must be sought. 


\section{NEMS SWITCH OPERATIONAL REQUIREMENTS}

Since NEMS relay topologies and much work on electrical switch contact behavior borrows from MEMS switches, it is useful to identify how the local contact conditions vary between the two technologies. MEMS relays have been used for power savings and superior functionality in RF systems [33], are now considered a mature technology, and have been commercially deployed. Many lessons learned for the contact behavior of conventional switch materials (i.e. metals) can be attributed to work focused on microscale, multiasperity MEMS contacts. Table 1 compares the contact environment and needs of NEMS and MEMS relays.

The lifetime requirements for NEMS logic switches differ substantially from RF MEMS switches, which are designed as interrupts for RF transmission lines. While these contacts carry RF signals up to $\mathrm{GHz}$ frequencies, making and breaking of the contact happens far less frequently, usually on the order of $\mathrm{kHz}$ or less, requiring $10^{8}-10^{11}$ cycles before failure in commercial applications. NEMS logic relays carry a DC signal and the interruption of that signal determines the clock frequency of the device. Thus, NEMS relays must exhibit nanosecond closure times, which requires upwards of $10^{16}$ operating cycles without failure [2].

\begin{tabular}{|l|l|l|}
\hline Requirement & MEMS RF Switches & NEMS Logic Switches \\
\hline Lifetime (cycles) & $10^{8}-10^{11}[33]$ & $10^{15}-10^{16}[2]$ \\
\hline True contact area & $10^{-8}-10^{-12} \mathrm{~m}^{2}$ & $10^{-17}-10^{-18} \mathrm{~m}^{2}$ \\
\hline Adhesion & $\begin{array}{l}\text { Not explicitly } \\
\text { treated }\end{array}$ & $\begin{array}{l}\text { Major concern (energy } \\
\text { use, reliability) [2] }\end{array}$ \\
\hline Actuation force & $\mu \mathrm{N}-\mathrm{mN}[33]$ & $5-150 \mathrm{nN}$ \\
\hline Voltage across contact & $2-70 \mathrm{~V}$ & Several $\mathrm{mV}$ to V \\
\hline Current through contact & $50-150 \mathrm{~mA}[33]$ & $\mathrm{nA}-\mu \mathrm{A}$ \\
\hline Current density & $10^{9}-10^{14} \mathrm{~A} / \mathrm{m}^{2}$ & $10^{5}-10^{11} \mathrm{~A} / \mathrm{m}^{2}$ \\
\hline Power & $<500 \mathrm{~mW}[33]$ & $<1 \mathrm{~mW}$ \\
\hline Max. contact resistance & $0.5-2 \Omega$ & $<10^{6} \Omega$ \\
\hline $\begin{array}{l}\text { Main contact materials } \Omega \\
\text { selection characteristics }\end{array}$ & $\begin{array}{l}\text { High conductivity and } \\
\text { non-fouling }\end{array}$ & $\begin{array}{l}\text { Moderate conductivity, } \\
\text { non-fouling, low adhesion }\end{array}$ \\
\hline
\end{tabular}

Table 1: Operational requirements of MEMS vs. NEMS switches.

The electrical constraints and power handling requirements of the electrical contacts of NEMS switches also differ substantially those of ohmic RF MEMS. Commercially viable ohmic RF MEMS switches demand contact resistances from $0.5-2 \Omega$ [33, p. 5] in order to minimize insertion loses, which limits possible set of contact materials to high conductivity metals. Permissible NEMS logic relay contact resistances have a broader range - from several $\mathrm{k} \Omega$ up to $100 \mathrm{k} \Omega$, depending on device topology and the electrical time constants of the implementation - such that alternative, novel, and previously unconsidered materials could be implemented at the contact. Furthermore, the power across ohmic RF MEMS contacts often reaches $100+\mathrm{mW}$ with power being transferred across the contact after closure (cold switching). NEMS logic relays are expected to experience, at most, a few $\mathrm{mW}$ of power across the contact and operate in a hot-switched mode (bias applied across the contact during closure) with $<1 \mathrm{~V}$ across the contacts during switching. These differing environments could change the degradation mechanisms. For instance, high voltages $(>10 \mathrm{~V})$ across MEMS contacts can lead to field evaporation, that may not be significant in nanoscale NEMS switch contacts.

\section{HIGH-THROUGHPUT MATERIALS ASSESSMENT}

We have developed a new high-throughput protocol developed for assessing the reliability of NEMS contact materials. An atomic force microscope (AFM) tip and a sample are each coated with the thin film materials of interest, using the same coating methods and conditions to be used for NEMS production. Tips are then imaged in scanning and transmission electron microscopes (SEM, TEM) to determine the quality of the coatings. TEM analysis requires no special sample preparation; the nano-scale tip diameter renders it sufficiently electron transparent (Figure 3). This approach has been tested by coating and characterizing tips with many different materials, e.g., $\mathrm{Pt}, \mathrm{Au}, \mathrm{Fe}$, amorphous carbon, diamond, and $\mathrm{Pt}_{\mathrm{x}} \mathrm{Si}$ (from annealing a $\mathrm{Pt} / \mathrm{Si}$ coating). Typical deposition times are only a few minutes and multiple tips can be coated in a single run for reproducibility tests.
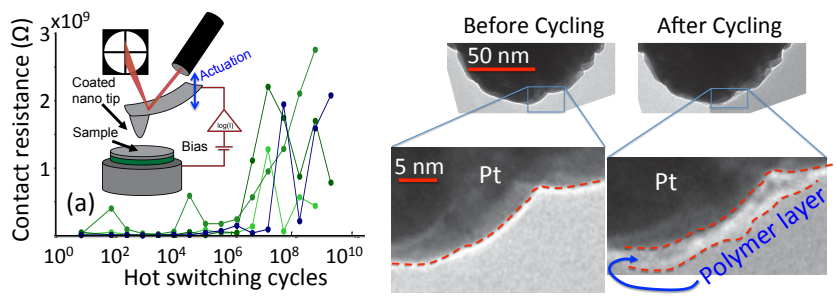

Figure 3: Left: High-throughput AFM test setup. High cycle testing of a series of Pt-Pt nanocontacts undergoing $5 \times 10^{9}$ test cycles in one day; failure (high resistance) is see at $\sim 10^{7}$ cycles. Inset: Dynamic AFM setup for high cycle testing. Right: TEM images of a Pt tip before and after $10^{9}$ hot switching tests against a Pt surface.

The cantilever is then inserted in the AFM and oscillated at high frequency to simulate a dynamically-contacting asperity within a NEMS interface (Figure 3). Contacts can be cycled with our without bias. The state of the interface is probed in situ by regularly interrupting the cycling to acquire current- and forcedisplacement measurements. These measurements can reveal signatures of TP, the role of contact area, and the tip-sample adhesion force. At present, the cantilever is oscillated at its resonance frequency, typically $\sim 50 \mathrm{kHz}$ which corresponds to $\sim 5 \times 10^{9}$ contact cycles in one day of testing. The right side of Figure 3 shows TEM images from before and after cycling a Pt tip against a Pt surface after $10^{9}$ hot switching cycles. Interestingly, nanoscale TP buildup is evident, while plastic deformation is undetectable, consistent with the resistance increase observed for the contact. Significant resistance increases in these tests are observed to occur at similar cycle numbers as those seen in actual NEMS switches, suggesting the method has promise for screening candidate NEMS contact materials, and for developing scientific understanding of failure.

\section{REFERENCES}

[1] D. A. Czaplewski, G. A. Patrizi, G. M. Kraus, J. R. Wendt, C. D. Nordquist, S. L. Wolfley, M. S. Baker, and M. P. de Boer, "A nanomechanical switch for integration with CMOS logic," J. Micromechanics Microengineering, vol. 19, no. 8, p. 085003, Aug. 2009.

[2] "International technology roadmap for semiconductors (2011 edition)." 2011.

[3] S. Borkar, "Exponential challenges, exponential rewards The future of moore's law," in Proceedings of the VLSISOC, Darmstadt, Germany, 2003.

[4] N. S. Kim, T. Austin, D. Baauw, T. Mudge, K. Flautner, J. S. Hu, M. J. Irwin, M. Kandemir, and V. Narayanan, "Leakage current: Moore's law meets static power," Computer, vol. 36, no. 12, pp. 68-75, 2003.

[5] N. Sinha, T. S. Jones, Z. Guo, and G. Piazza, "Body-biased complementary logic implemented using aln piezoelectric MEMS switches," J. Microelectromechanical Syst., vol. 21, 
no. 2, pp. 484-496, 2012.

[6] U. Zaghloul and G. Piazza, "10 - $25 \mathrm{~nm}$ piezoelectric nanoactuators and NEMS switches for millivolt computational logic," in IEEE 26th International Conference on Micro Electro Mechanical Systems (MEMS), Taipei, Taiwan, 2013, pp. 233-236.

[7] R. Nathanael, V. Pott, H. Kam, J. Jeon, and T.-J. King Liu, "4-terminal relay technology for complementary logic," in 2009 IEEE International Electron Devices Meeting (IEDM 2009), Piscataway, NJ, USA, 2009.

[8] M. Tabib-Azar, S. R. Venumbaka, K. Alzoubi, and D. Saab, "1 volt, $1 \mathrm{GHz}$ NEMS switches," presented at the IEEE Sensors, 2010, pp. 1424-1426.

[9] W. W. Jang, J.-B. Yoon, M.-S. Kim, J.-M. Lee, S.-M. Kim, E.-J. Yoon, K. H. Cho, S.-Y. Lee, I.-H. Choi, D.-W. Kim, and D. Park, "NEMS switch with $30 \mathrm{~nm}$-thick beam and $20 \mathrm{~nm}$-thick air-gap for high density non-volatile memory applications," Solid-State Electron., vol. 52, no. 10, pp. 1578-1583, Oct. 2008.

[10] O. Y. Loh and H. D. Espinosa, "Nanoelectromechanical contact switches," Nat. Nanotechnol., vol. 7, no. 5, pp. 283295, 2012.

[11] J. D. Meindl, Q. Chen, and J. A. Davis, "Limits on silicon nanoelectronics for terascale integration," Science, vol. 293, no. 5537, pp. 2044-2049, Sep. 2001.

[12] J. A. . Hutchby, G. I. . Bourianoff, V. V. . Zhirnov, and J. E. . Brewer, "Extending the road beyond CMOS," IEEE Circuits Devices Mag., vol. 18, no. 2, pp. 28-41, Mar. 2002.

[13] F. Chen, H. Kam, D. Markovic, T. J. K. Liu, V. Stojanovic, and E. Alon, "Integrated circuit design with NEM relays," in Proceedings of the 2008 IEEE/ACM International Conference on Computer-Aided Design, 2008, pp. 750-757.

[14] D. A. Czaplewski, G. M. Kraus, and C. D. Nordquist, "Nanomechanical switches for power saving in CMOS applications," Electron. Lett., vol. 45, no. 11, pp. 550-551, 2009.

[15] V. Pott, Hei Kam, R. Nathanael, Jaeseok Jeon, E. Alon, and Tsu-Jae King Liu, "Mechanical computing redux: Relays for integrated circuit applications," Proc. IEEE, vol. 98, no. 12, pp. 2076-2094, Dec. 2010.

[16] M. Spencer, F. Chen, C. C. Wang, R. Nathanael, H. Fariborzi, A. Gupta, H. Kam, V. Pott, J. Jeon, T.-J. K. Liu, D. Markovic, E. Alon, and V. Stojanovic, "Demonstration of integrated micro-electro-mechanical relay circuits for VLSI applications," IEEE J. Solid-State Circuits, vol. 46, no. 1, pp. 308-320, Jan. 2011.

[17] H. F. Dadgour and K. Banerjee, "Hybrid NEMS-CMOS integrated circuits: a novel strategy for energy-efficient designs," IET Comput. Digit. Tech., vol. 3, no. 6, p. 593, 2009.

[18] K. Akarvardar, D. Elata, R. Parsa, G. C. Wan, K. Yoo, J. Provine, P. Peumans, R. T. Howe, and H.-S. Wong, "Design considerations for complementary nanoelectromechanical logic gates," in Electron Devices Meeting, 2007. IEDM 2007. IEEE International, 2007, pp. 299-302.

[19] A. M. Ionescu, V. Pott, R. Fritschi, K. Banerjee, M. J. Declercq, P. Renaud, C. Hibert, P. Fluckiger, and G. A. Racine, "Modeling and design of a low-voltage SOI suspended-gate MOSFET (SG-MOSFET) with a metal-over-gate architecture," in Quality Electronic Design, 2002. Proceedings. International Symposium on, 2002, pp. 496-501.

[20] F. Fallah and M. Pedram, "Standby and active leakage current control and minimization in cmos vlsi circuits," IEICE Trans. Electron., vol. E88-C, no. 4, pp. 509-519, Apr. 2005.

[21] E. Yablonovitch, "There are orders-of-magnitude power advantages in complementing the transistor with a milli-volt switch," presented at the MTO Symposium, San Jose, CA, USA, 07-Mar-2007.

[22] N. Abele, R. Fritschi, K. Boucart, F. Casset, P. Ancey, and A.-M. Ionescu, "Suspended-gate MOSFET: bringing new MEMS functionality into solid-state MOS transistor," in Electron Devices Meeting, 2005. IEDM Technical Digest. IEEE International, 2005, pp. 479-481.

[23] S.-W. Lee, R. W. Johnstone, and A. M. Parameswaran, "MEMS mechanical logic units: characterization and improvements of devices fabricated with MicraGEM and PolyMUMPs," 2005, p. 60371A-60371A-10.

[24] T.-H. Lee, S. Bhunia, and M. Mehregany, "Electromechanical computing at $500 \mathrm{C}$ with silicon carbide," Science, vol. 329, no. 5997, pp. 1316-1318, Sep. 2010.

[25] C. Chen, R. Parsa, N. Patil, S. Chong, K. Akarvardar, J. Provine, D. Lewis, J. Watt, R. T. Howe, H. S. P. Wong, and others, "Efficient FPGAs using nanoelectromechanical relays," in Proceedings of the 18th Annual Acm/Sigda International Symposium on Field Programmable Gate Arrays, 2010, pp. 273-282.

[26] Y. Zhou, S. Thekkel, and S. Bhunia, "Low power FPGA design using hybrid CMOS-NEMS approach," in Proceedings of the 2007 international symposium on Low power electronics and design, 2007, pp. 14-19.

[27] W. Y. Choi, Hei Kam, D. Lee, J. Lai, and T.-J. King Liu, "Compact nano-electro-mechanical non-volatile memory (NEMory) for 3D integration," in 2007 IEEE International Electron Devices Meeting (IEDM 2007), Washington, DC, USA, 2007, pp. 603-6.

[28] S. Fujita, K. Nomura, K. Abe, and T. H. Lee, "3-d nanoarchitectures with carbon nanotube mechanical switches for future on-chip network beyond cmos architecture," IEEE Trans. Circuits Syst. Regul. Pap., vol. 54, no. 11, pp. 24722479, Nov. 2007.

[29] L. Chen, H. Lee, Z. J. Guo, N. E. McGruer, K. W. Gilbert, S. Mall, K. D. Leedy, and G. G. Adams, "Contact resistance study of noble metals and alloy films using a scanning probe microscope test station," J. Appl. Phys., vol. 102, no. 7, pp. 074910-1, Oct. 2007.

[30] D. A. Czaplewski, C. D. Nordquist, C. W. Dyck, G. A. Patrizi, G. M. Kraus, and W. D. Cowan, "Lifetime limitations of ohmic, contacting RF MEMS switches with $\mathrm{Au}, \mathrm{Pt}$ and Ir contact materials due to accumulation of 'friction polymer' on the contacts," J. Micromechanics Microengineering, vol. 22, no. 10, p. 105005, Oct. 2012.

[31] R. A. Coutu Jr., P. E. Kladitis, K. D. Leedy, and R. L. Crane, "Selecting metal alloy electric contact materials for MEMS switches," J. Micromechanics Microengineering, vol. 14, no. 8, pp. 1157-1164, 2004.

[32] V. Brand, M. S. Baker, and M. P. de Boer, "Contamination Thresholds of Pt- and -Coated Ohmic Switches," J. Microelectromechanical Syst., vol. 22, no. 6, pp. 1248-1250, Dec. 2013.

[33] G. M. Rebeiz, RF MEMS : theory, design, and technology. Hoboken, NJ: J. Wiley, 2003.

\section{CONTACT}

*R.W. Carpick, tel: +1-215-898-4608; carpick@seas.upenn.edu 